Pacific Journal of Mathematic 


\title{
COHOMOLOGY OF DIAGRAMS AND EQUIVARIANT SINGULAR THEORY
}

\author{
R. J. Piacenza
}

\begin{abstract}
The purpose of this paper is to define a cohomology theory for diagrams of simplicial sets that specializes to Illman's equivariant singular cohomology for discrete $G$. We show that such a theory is representable by a suitable Eilenberg-Maclane object. The paper concludes with a comparison of equivariant singular cohomology and equivariant sheaf cohomology.

We adopt the category theory of Maclane as formulated in "Categories for the working mathematician" and use the framework of Quillen's "Homotopical Algebra."
\end{abstract}

I. Preliminaries. We let $\Delta$ be the category of finite ordered sets and $S S$ the category of simplicial sets as in [11]. If $A$ is any category $c A$ will denote the category of cosimplicial objects in $A$, i.e., $c A=$ Funct $(\Delta, A)$.

If $J$ is a small category $J S$ denotes the small complete and cocomplete functor category Funct $\left(J^{o p}, S S\right)$ and $J A$ the category of abelian group objects in $J S$. Furthermore, if $F \in J S$ and $K \in S S$ we define $F \otimes K$ and $F^{K}$ pointwise by $F \otimes K(j)=F(j) \times K$ and $F^{K}(j)=$ $F(j)^{K}$.

$J S$ may be enriched in $S S$ by the functor Nat: $J S^{o p} \times J S \rightarrow S S$ defined by $\operatorname{Nat}(E, F)_{n}=\operatorname{Nat}(E \otimes \Delta[n], F)$ where $\Delta[n]$ is the standard $n$-simplex in $S S$. Thus $J S$ is a simplicial category in the sense of [14], Chapter II. We note that $J S$ is tensored over $S S$ via ( ) $\otimes K$ and cotensored over $S S$ via ()$^{K}$.

A strict homotopy is a morphism of the form $F \otimes \Delta[1] \rightarrow E$ and gives rise to the strict homotopy relation on morphisms of JS. We let the homotopy relation on morphisms of $J S$ be the equivalence relation generated by the strict homotopy relation. We denote the homotopy category of $J S$ by $h J S$ with Hom sets $h N a t(E, F)$ abbreviated $h(E, F)$.

A morphism $f: E \rightarrow F$ is called a fibration, respectively weak equivalence, if $f(j)$ is a fibration, respectively weak equivalence, for each $j \in J$. A cofibration is a morphism that has the left lifting property with respect to all trivial fibrations. We have the following result of Quillen-Bousfield-Kan [1], pg. 313:

THEOREM 1.1. JS equipped as above is a closed simplicial model category. 
We let HoJS denote the localization of $J S$ at the weak equivalences as in [14]. Note that if $E \in J S$ is cofibrant and $F$ is fibrant then $h(E, F)=H o J S(E, F),[14]$, Chapter I, Corollary 1, 1.16.

II. Cohomology theory. Let $A b_{*}$ be the category of nonnegative chain complexes of abelian groups with chain maps as morphisms and $L: S S \rightarrow A b_{*}$ the free chain complex functor as in [11], pg. 5 .

By a covariant system of coefficients on $J$ we mean a covariant functor $\Pi: J \rightarrow A b$. Fixing a covariant system $\Pi$ and $F \in J S$ there are functors $J^{o p} \times J \stackrel{F \times \Pi}{\longrightarrow} S S \times A b \stackrel{L \times 1}{\longrightarrow} A b_{*} \times A b \stackrel{\otimes}{\longrightarrow} A b_{*}$ and thus a composite functor $F_{I I}: J^{o p} \times J \rightarrow A b_{*}$. Define $C_{*}(F ; \Pi)$ as the coend of the functor $F_{\Pi}$, denoted $C_{*}(F ; \Pi)=\int^{j} F_{\Pi}(j, j)$. Let $H_{*}(F ; \Pi)$ be the associated homology, i.e., $H_{n}(F ; \Pi)=H_{n}\left[C_{n}(F ; \Pi)\right]$. Clearly $C_{*}$ and $H_{*}$ are natural in both variables.

By a contravariant system of coefficients on $J$ we mean a functor $\Pi: J^{o p} \rightarrow A b$. Fixing a contravariant system $I I$ and $F \in J S$ we have $J \times J^{o p} \stackrel{F^{o p} \times \Pi}{\longrightarrow} S S^{o p} \times A b \stackrel{L^{o p} \times 1}{\longrightarrow} A b_{*}^{o p} \times A b \stackrel{\text { Hom }}{\longrightarrow} A b^{*}$ where $A b^{*}$ is the category of cochain complexes. We let $F^{\Pi}: J \times J^{o p} \rightarrow A b^{*}$ be the composite of the above functors. Define $C^{*}(F ; I)$ as the end of the functor $F^{I}$, denoted $\int_{j} F^{\Pi}(j, j)$. Let $H^{*}(F ; \Pi)$ be the associated cohomology, i.e., $H^{n}\left(F_{j} ; \Pi\right)=H^{n}\left[C^{*}(F ; \Pi)\right]$. Clearly $C^{*}$ aud $H^{*}$ are natural in both variables, contravariant in the first and covariant in the second. $H^{*}(; \Pi)$ gives rise to a cohomology theory on $J S$ called singular cohomology with coefficients $\Pi$.

For the remainder of this paper we restrict our attention to cohomology theories.

We start by giving an explicit description of $C^{n}(F ; \Pi)$ and its coboundary $\delta^{n}$. A cochain $\phi \in C^{n}(F ; \Pi)$ is a family of functions $\phi_{j}: F(j)_{n} \rightarrow \Pi(j), \quad j \in J$ satisfying for each $f: i \rightarrow j, \Pi(f) \phi_{j}=\phi_{i} F(f)$. $\delta^{n} \phi \in C^{n+1}(F ; \Pi)$ is defined by $\delta \phi_{j}=\sum_{k}(-1)^{k} \phi_{j} \partial_{k}$ where $\partial_{k}$ is the $k$ th face operator. We let $Z^{n}(F ; \Pi)$ be the group of $n$-dimensional cocycles, i.e., $\operatorname{Ker}\left(\partial^{n}\right)$.

Let $i: F \rightarrow E$ be an inclusion. One may easily check $C^{*}(i)$ : $C^{*}(E ; \Pi) \rightarrow C^{*}(F ; \Pi)$ is an epimorphism. We define $C^{*}(E, F ; \Pi)=$ $\operatorname{Ker} C^{*}(i)$ and relative cohomology by $H^{n}(E, F ; \Pi)=H^{n}\left[C^{*}(E, F ; \Pi)\right]$.

We omit the proofs of the following four propositions as they are standard.

Proposition 2.1. Each inclusion $F \rightarrow E$ induces a long exact sequence in cohomology.

Proposition 2.2. If $f, g$ are homotopic in JS then $H^{*}(f)=H^{*}(g)$. 
Proposition 2.3. If $E=D \cup F$ then the inclusion $(D, F \cap D) \rightarrow$ $(E, F)$ induces an isomorphism in cohomology.

Proposition 2.4. If $E=\frac{1}{\lambda \in \Lambda} E_{\lambda}$ then $H^{*}(E) \cong \Pi_{\lambda} H^{*}\left(E_{\lambda}\right)$.

Let $j \in J$. Define $M_{j} \in J S$ by $M_{j}(i)_{n}=J(i, j)$ with identities as face and degeneracy maps. One may use Yoneda's theorem to show:

Proposition 2.5. $H^{n}\left(M_{j} ; \Pi\right)=0$ if $n>0$ and $H^{\circ}\left(M_{j} ; \Pi\right)=\Pi(j)$.

Now let $\Pi$ be a fixed contravariant coefficient system and $K(\Pi, n)$ the object of $J A$ formed by composing $\Pi$ with $K(, n): A b \rightarrow S A$, i.e., the $n$th Eilenberg-Maclane functor as in [11], $\$ 23$, pg. 101. Using the explicit description of cochains the results of [11], $\S 24$ generalize to the following theorem.

THEOREM 2.6. There are natural isomorphisms of group valued functors $Z^{n}(; \Pi) \cong \operatorname{Nat}(; K(\Pi, n))$ and $H^{n}(; \Pi) \cong h(, K(\Pi, n))$ for all $n \geqq 0$.

There is an obvious free abelianization functor $L_{a b}: J S \rightarrow J A$ and we are in the situation of [14], Chapter 2, $\$ 5$. Clearly we have:

Corollary 2.7. If $E$ is cofibrant than $H^{*}(E ; \Pi)$ coincides with Quillen's homotopical cohomology as defined in [14] Chapter II, 5.1 .

We close this section with two examples.

EXAMPLE 2.8. Let $D: J \rightarrow c$ Top be a functor where Top is the category of compactly generated spaces in the sense of [16]. For example $D$ could be a diagram of generalized intervals in the sense of [13]. Define a functor Top $\rightarrow J S$ by $X \mapsto \bar{X}=\operatorname{Top}(D(), X)$ and $D$-singular cohomology by $H_{j}^{*}(X ; \Pi)=H^{*}(\bar{X} ; \Pi)$. We observe that by $2.2 H_{\hat{\nu}}^{*}$ satisfies the $D$-homotopy axiom, i.e., $f, g$ in Top are $D$-homotopic if $\bar{f}, \bar{g}$ are homotopic in $J S$.

ExAmple 2.9. Let $M \in S S$. Define $M^{\prime} \in J S$ to be the constant diagram with value $M$, i.e., $M^{\prime}(j)=M$ and $M^{\prime}(f)=1_{M}$. From our description of cochains we have $C^{*}\left(M^{\prime} ; \Pi\right) \cong C^{*}(M$, lim $\Pi)$ where the right side is the ordinary cochain complex of $M$ with coefficients in the abelian group $\lim \Pi$. One may also check that $K(\lim \Pi, n) \cong$ $\lim K(\Pi, n)$. Compare with [1], Chapter XI iii, pg. 288. 
III. Equivariant singular theory. Throughout this section $G$ is a fixed group, discrete unless specified otherwise. G-Set is the category of left $G$-sets and $J$ is the full subcategory of $G$-Set determined by $G / H$ as $H$ varies over all subgroups of $G$.

By a $G$-complex we mean a simplicial set with $G$ acting on the left as automorphisms. We let $G$-SS be the category of left $G$-complexes with the obvious morphisms. If $X \in G-S S$ and $K \in S S$ we may form $X \otimes K \in G-S S$ by taking $X \times K$ with $G$ acting in the left coordinate. Thus G-SS is tensored over SS.

We denote by $G$-Top the category of left $G$-spaces.

If $X$ is in any of the above categories and $H$ is a subgroup of $G$ we let $X^{H}=\{x \in X \mid h x=x$ for all $h \in H\}$. We note the following adjunctions: $G-S S(G / H \otimes K, X) \cong S S\left(K, X^{H}\right)$ and $G-T o p(G / H \times K, X) \cong$ $\operatorname{Top}\left(K, X^{H}\right)$ where $G / H \times K$ has $G$ acting in the left coordinate only.

We define functors $I: G-S S \rightarrow J S$ by $I(X)(G / H)=G$-Set $(G / H, X)=$ $X^{H} \in S S$ and $T: J S \rightarrow G-S S$ by $T(F)=F(G)$ provided with its natural $G$-action acquired from $G$-Set $(G, G)=G$.

Let $f: T(F) \rightarrow X$ be a morphism in G-SS. Define $f^{\prime}: F \rightarrow I(X)$ by $f^{\prime}(\sigma)=f F(q)(\sigma)$ for $\sigma \in F(G / H)_{n}$ and $q: G \rightarrow G / H$ the natural quotient map. It is routine to check that $f^{\prime}$ is natural.

Furthermore if $h ; F \rightarrow I(X)$ then $h(\sigma)=h^{\sharp} F(q)(\sigma)$ where $h^{\sharp}: F(G) \rightarrow X$ is the G-component of $h$, i.e., $h$ is determined by $h^{\sharp}$. We have thus established:

Proposition 3.1. I is full and faithful and right adjoint to $T$. Furthermore $T$ preserves limits and both $T$ and $I$ preserve tensor products over SS.

Using $I$ we view $G-S S$ as a subcategory of $J S$.

A morphism $f: E \rightarrow F$ of $G-S S$ is said to be a fibration, respectively weak equivalence if $I(f)$ is a fibration, respectively weak equivalence of JS. A cofibration in G-SS is a morphism of G-SS that has the left lifting property for all trivial fibrations in G-SS. We have:

Proposition 3.2. G-SS equipped as above is a closed model category. Furthermore each monomorphism of G-SS is a cofibration and thus any object of G-SS is cofibrant.

Proof. G-SS is the category of simplicial objects in G-Set hence a closed model category by [14] Chapter II, Theorem 4. The second assertion follows from a simple lifting argument and induction over the skeletons.

We note that for $E \in G-S S, I(E)$ may not be cofibrant in $J S$. 
Consider the adjoint pair $U:$ Top $\rightarrow S S$ where $U(X)$ is the singular complex of $X$ and | $: S S \rightarrow$ Top is the geometric realization (see [7], [11], and [17], pg. 36). These functors yield by naturality an adjoint pair $U_{G}: G$-Top $\rightarrow G$-SS and ||$_{G}: G-S S \rightarrow G$-Top with natural isomorphism $G-\operatorname{Top}\left(|F|_{G}, X\right) \cong G-S S\left(F, U_{G}(X)\right)$. We note by [17], Lemma 3.2.4, pg. 40 that $|F|_{G}$ is a $G-C W$ complex in the sense of [17], pg. 10; see also [2].

Proposition 3.3. The counit of the above adjunction $\psi:\left|U_{G} X\right|_{G} \rightarrow$ $X$ is a weak G-equivalence.

Proof. $\psi^{H}:\left|U_{G} X\right|_{G}^{H} \rightarrow X^{H}$ is just the counit $\left|U X^{H}\right| \rightarrow X^{H}$ and thus a weak equivalence by [11], Thm. 16.6, pg. 65 .

Corollary 3.4. If $X$ is a G-CW complex then $\psi$ is an equivariant homotopy equivalence.

Proof. A direct application of 3.3 and [17], Corollary 1.3.4, pg. 12.

Let $H o G-S S$ be G-SS localized at the weak equivalences and $H o G$-Top be $G$-Top localized at the weak $G$-equivalences.

Proposition 3.5. $U_{G}$ and ||$_{G}$ preserve weak equiavlances and induce an equivalence of categories $H o G-T o p \cong H o G-S S$.

Proof. Follows from the Adjoint functor lemma of [4], pg. 426 together with 3.3.

CoRollary 3.6. Let $h G-K S$ be the homotopy category of fibrant objects in G-SS and $h G-C W$ the equivariant homotopy category of $G-C W$ complexes then $U_{G}$ and ||$_{G}$ induce an equivalence of categories $h G-K S \cong h G-C W$.

We define equivariant singular cohomology as follows: Let $\Delta_{*}$ be the standard cosimplicial space with $\Delta_{n}$ the topological $n$-simplex. Define a functor $D: J \rightarrow c G$-Top by $D(G / H)=G / H \times \Delta_{*}$ with $G$ acting in the left coordinate and $D(f)=f \times 1$ for $f$ a morphism of $J$. As in Example 2.8 we have a functor $G$-Top $\rightarrow J S$ defined by $X \rightarrow \bar{X}=$ $G-\operatorname{Top}(D(), X)$. We define $C_{\sigma}^{*}(X ; \Pi)$ by $C_{G}^{*}(X ; \Pi)=C^{*}(\bar{X} ; \Pi)$ and equivariant cohomology by $H_{G}^{*}(X ; \Pi)=H^{*}(\bar{X} ; \Pi)$. Because $G$ is discrete an equivariant map $T: G / H \times \Delta_{n} \rightarrow G / K \times \Delta_{n}$ covering the identity of $\Delta_{n}$ is of the form $f \times 1$ for a unique equivariant map $f: G / H \rightarrow$ $G / K$. This implies: 
Proposition 3.7. $H_{G}^{*}(; I)$ is isomorphic to Illman's equivariant singular cohomology as defined in [8] and [9].

In [9] Illman shows that equivariant singular cohomology satisfies all the equivariant Eilenberg-Steenrod axioms. In addition we have in our setting:

Proposition 3.8. The theory $H_{G}^{*}(; \Pi)$ satisfies the wedge axiom and the strong homotopy axiom, i.e., if $f: X \rightarrow Y$ is a weak equivalence in G-Top then $H_{G}^{*}(f)$ is an isomorphism.

Proof. 2.4 implies the wedge axiom. Because $\bar{f}: \bar{X} \rightarrow \bar{Y}$ is a weak equivalence in $G-S S$ and $\bar{X}, \bar{Y}$ are both fibrant and cofibrant in G-SS, $f$ is a homotopy equivalence by results of [14]. The result then follows from 2.2 .

As in [14] Chapter II, 5.3, Example 1, we have for $I I$ a left $G$-module that $H_{G}^{*}(; I I)$ is isomorphic on G-SS to Quillen's homotopical cohomology. The relationship between $H^{*}(; \Pi)$ and homotopical cohomology in general is unclear. (When is $\operatorname{HoJS}(E, K(\Pi, n))=$ $h J S(E, K(\Pi, n))$ ?) We leave this an as open question.

If $G$ is only a semigroup much of what we have done may be carried through by replacing $J$ by a small subcategory of $G$-set. We leave this for the reader.

Before going further we point out a close relationship between equivariant cohomology and prestack cohomology, as defined in [5] and [6].

Let $F \in G$-SS with natural projection $p: F \rightarrow F / G$. Let $\Pi$ be a fixed contravariant coefficient system. Note that $F=\Perp_{x \in F^{\prime} G} p^{-1}(x)$. Define a prestack $\check{I}$ of abelian groups on $F / G$ as follows: If $\sigma \in$ $(F / G)_{n}, \check{\Pi}(\sigma)=\operatorname{Nat}\left(p^{-1}(\sigma), \Pi\right)$ where the $G$-set $p^{-1}(\sigma)$ is viewed as a functor $J^{o p} \rightarrow$ Set. Now a face map $\partial$ of $F / G$ gives an equivariant map $p^{-1}(\sigma) \rightarrow p^{-1}(\partial \sigma)$ and thus a natural homomorphism $\check{\Pi}(\partial): \Pi(\partial \sigma) \rightarrow$ $\check{\Pi}(\sigma)$.

Now there is clearly an isomorphism of cochain complexes $f: C^{*}(F ; \Pi) \rightarrow \bar{C}^{*}(F / G ; \Pi)$ where the right side is the complex defined in [6], pg. 602. Thus we have an isomorphism of equivariant cohomology with prestack cohomology.

The isomorphism $f$ is the simplicial analogue of Eilenberg's classical result relating the equivariant cohomology of the universal cover of a complex to local cohomology. See [18] Chapter VI, Thm. $3.4^{*}$.

We now generalize the classical result of Eilenberg by replacing a universal covering space by a $G$ space and a left $G$-module by a coefficient system $\Pi$. We formulate this result for any topological 
group $G$.

Let $G$ be a topological group and $\mathscr{F}$ an orbit family for $G$ as in [9], pg. 3. Let $J$ be the full subcategory of G-Top with objects $G / H$ with $H \in \mathscr{F}$. A contravariant coefficient system $\Pi$ on $J$ is said to be homotopy invariant if $\Pi(f)=\Pi(g)$ whenever $f, g: G / H \rightarrow$ $G / K$ are homotopic by an equivariant homotopy.

Let $X \in G$-Top and $I I$ a homotopy invariant coefficient system. We let $I_{G}^{n}(X ; \Pi)$ be the group of equivariant singular cochains as defined by Illman in [9] Def. 4.3, pg. 21. Note that $I_{G}^{*}(X ; \Pi) \subseteq$ $C^{*}(\bar{X} ; \Pi)$ where $\bar{X}$ is defined just as before. Observe that $I_{G}^{*}(X ; \Pi)=$ $C^{*}(\bar{X} ; \Pi)$ if $G$ is discrete and $\mathscr{F}$ is the set of all subgroups of $G$. We let $H_{G}^{*}(X ; \Pi)$ be the cohomology of $I_{G}^{*}(X ; \Pi)$ which is in general distinct from $H^{*}(\bar{X} ; \Pi)$.

Let $\Pi$ be a fixed homotopy invariant coefficient system on $J$ and $X \in G$-Top with its natural quotient $q: X \rightarrow X / G$.

Define a cochain complex of presheaves $S^{*}$ over $X / G$ by setting $S^{n}(U)=I_{G}^{n}\left(q^{-1}(U) ; \Pi\right)$ with its natural coboundary. Note that $S^{n}$ for all $n \geqq 0$ satisfies condition $S_{2}$ of [3] pg. 6 and $S^{0}$ is a sheaf. Thus we obtain a short exact sequence of complexes $0 \rightarrow S_{0}^{*} \rightarrow S^{*} \rightarrow$ $\mathscr{S}^{*} \rightarrow 0$ where $\mathscr{S}^{n}$ is $S^{n}$ sheafied and $S^{*} \rightarrow \mathscr{S}^{*}$ is the natural epimorphism. (Compare [3] pg. 19.)

Proposition 3.9. For each $n \geqq 0 S^{n}$ is a fine presheaf in the sense of [15] pg. 330 .

Proof. The argument of [15] Example 2, pg. 330 may easily be adapted.

Now consider the cochain complex $S_{0}^{*}(U)$ of locally zero cochains on an open set $U \subseteq X / G$. We may use [9] Proposition 6.4, pg. 35 to show:

Proposition 3.10. $S_{0}^{*}(U)$ is an acyclic complex.

Let $\widetilde{\Pi}$ be the presheaf on $X / G$ defined by the formulae: $\tilde{\Pi}(U)=$ $H_{G}^{0}\left(q^{-1}(U) ; \Pi\right)$. Using a simple subdivision argument one may easily check that $\widetilde{\Pi}$ is a sheaf. Furthermore the sequence $0 \rightarrow \widetilde{\Pi} \rightarrow \mathscr{S}^{0} \stackrel{\tilde{\delta}^{0}}{\rightarrow}$ $\mathscr{S}^{1}$ is exact, i.e., $\widetilde{\Pi}=\operatorname{Ker}\left(\delta^{0}\right)$.

An $X \in G$-Top is said to be $G$ cohomologically locally connected, abbreviated $G$-cle if the complex of sheaves $0 \rightarrow \widetilde{I} \rightarrow \mathscr{S}^{*}$ is exact for any homotopy invariant system $\Pi$.

Consider the following conditions on $X \in G$-Top.

(i) For each $x \in X / G$ the orbit $q^{-1}(x)$ is isomorphic to $G / H$ for some $H \in \mathscr{F}$. 
(ii) $X$ is G-locally contractable, i.e., each orbit $q^{-1}(x)$ is an equivariant neighborhood deformation retract of an arbitrarily small $G$-invariant neighborhood.

If $X \in G$-Top satisfies (i) and (ii) then $X$ is G-clc. This follows from the dimension axiom and homotopy axiom for equivariant singular cohomology. (See [9].)

We now have the comparison theorem:

THEOREM 3.11. If $X \in G$-Top is G-clc with $X / G$ paracompact then there is a natural isomorphism $H_{G}^{*}(X ; \Pi) \cong H^{*}(X / G, \widetilde{I})$ where the right side is sheaf cohomology and $\Pi$ is any homotopy invariant coefficient system.

Proof. By $3.10 S_{0}^{*}$ is an acyclic complex and by $3.9 \mathscr{S}^{n}$ is a fine sheaf in the sense of [3]. Therefore $0 \rightarrow \widetilde{I} \rightarrow \mathscr{S}^{*}$ is a fine resolution of $\widetilde{\Pi}$ and the result follows.

The proof of the following corollaries are left to the reader.

Corollary 3.12. Suppose $X \in G$-Top satisfies the assumptions of 3.11. If $\operatorname{dim}(X / G) \leqq n$ then $H_{G}^{k}(X ; \Pi)=0$ for all $k>n$ (dim is defined as in [3], pg. 73).

Compare 3.12 with [18], Chapter VI, Corollary 4.2.

CoROllary 3.13. Let $G$ be discrete and $X$ a principal $G$ bundle with $X / G$ paracompact and locally contractable. If $M$ is a left G-module then $H_{G}^{*}(X ; M) \cong H^{*}(X / G ; \widetilde{M})$ where $\widetilde{M}$ is the locally trivial sheaf on $X / G$ determined by $X$ and $M$.

Compare 3.13 with [18], Chapter VI, Thm. 3.4.*

Finally I would like to thank Professor M. Mielke for his many helpful suggestions.

\section{REFERENCES}

1. A. K. Bousfield and D.M. Kan, Homotopy Limits, Completions and Localizations, Springer Notes \#304, 1972.

2. G. Bredon, Equivariant Cohomology Theories, Springer Notes \#34, 1967.

3. - Sheaf Theory, McGraw-Hill, 1967.

4. K. S. Brown, Homotopy Theory and Sheaf Cohomology, Trans. Amer. Math. Soc., 186 (1973), 419-458.

5. Y. C. Chen, Stacks, co-stacks and axiomatic homology theory, Trans. Amer. Math. Soc., 145 (1969), 105-116.

6. - On Spanier's higher order operations, Proc. Amer. Math. Soc., 31 (1972), 601-604. 
7. P. Gabriel and M. Zisman, Calculus of Fractions and Homotopy Theory, SpringerVerlag, 1967.

8. S. Illman, Equivariant singular homology and cohomology, Bull. Amer. Math. Soc., 79 (1973), 188-192.

9. ——, Equivariant singular homology and cohomology $I$, Memoir of the Amer. Math. Soc., \#156, (1975).

10. S. Maclane, Categories for the Working Mathematician, Springer-Verlag, 1971.

11. P. May, Simplicial Objects in Algebraic Topology, Van Nostrand Studies, \#11, 1967.

12. M. Mielke, Equivariant and hyperequivariant cohomology, Illinois J. Math., 20 (1976), 177-185.

13. - The interval in algebraic topology, to appear.

14. D. Quillen, Homotopical Algebra, Springer Notes, \#43, 1967.

15. E. Spanier, Algebraic Topology, McGraw-Hill, 1966.

16. R. M. Vogt, Convenient categories of topological spaces for homotopy theory, Arch. Math., 22 (1971), 545-555.

17. S. Waner, Equivariant Classifying Spaces and Fibrations, Thesis, University of Chicago, 1978.

18. G. W. Whitehead, Elements of Homotopy Theory, Springer-Verlag, 1978.

Received October 2, 1979 and in revised form July 9, 1980.

UNIVERSITY OF ALASKa

FAIRBANKS, AK 99701 



\section{PACIFIC JOURNAL OF MATHEMATICS}

\section{EDITORS}

DONALD BABBITT (Managing Editor)

University of California

Los Angeles, CA 90024

Hugo RossI

University of Utah

Salt Lake City, UT 84112

C. C. MOORE and ANDREW OGG

University of California

Berkeley, CA 94720

\section{J. DugundjI}

Department of Mathematics

University of Southern California

Los Angeles, CA 90007

R. FinN and J. Milgram

Stanford University

Stanford, CA 94305

\section{ASSOCIATE EDITORS}
E. F. BeCKENBACH
B. H. Neumann
F. WoLF
K. YoSHIDA

\section{SUPPORTING INSTITUTIONS}

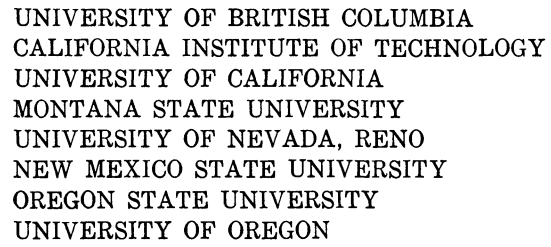

UNIVERSITY OF BRITISH COLUMBIA CALIFORNIA INSTITUTE OF TECHNOLOGY UNIVERSITY OF CALIFORNIA MONTANA STATE UNIVERSITY UNIVERSITY OF NEVADA, RENO NEW MEXICO STATE UNIVERSITY OREGON STATE UNIVERSITY UNIVERSITY OF OREGON

\author{
UNIVERSITY OF SOUTHERN CALIFORNIA \\ STANFORD UNIVERSITY \\ UNIVERSITY OF HAWAII \\ UNIVERSITY OF TOKYO \\ UNIVERSITY OF UTAH \\ WASHINGTON STATE UNIVERSITY \\ UNIVERSITY OF WASHINGTON
}

The Supporting Institutions listed above contribute to the cost of publication of this Journal, but they are not owners or publishers and have no responsibility for its content or policies.

Mathematical papers intended for publication in the Pacific Journal of Mathematics should be in typed form or offset-reproduced, (not dittoed), double spaced with large margins. Please do not use built up fractions in the text of the manuscript. However, you may use them in the displayed equations. Underline Greek letters in red, German in green, and script in blue. The first paragraph or two must be capable of being used separately as a synopsis of the entire paper. Please propose a heading for the odd numbered pages of less than 35 characters. Manuscripts, in triplicate, may be sent to any one of the editors. Please classify according to the scheme of Math. Reviews, Index to Vol. 39. Supply name and address of author to whom proofs should be sent. All other communications should be addressed to the managing editor, or Elaine Barth, University of California, Los Angeles, California, 90024.

50 reprints to each author are provided free for each article, only if page charges have been substantially paid. Additional copies may be obtained at cost in multiples of 50 .

The Pacific Journal of Mathematics is issued monthly as of January 1966. Regular subscription rate: $\$ 84.00$ a year (6 Vols., 12 issues). Special rate: $\$ 42.00$ a year to individual members of supporting institutions.

Subscriptions, orders for numbers issued in the last three calendar years, and changes of address shoud be sent to Pacific Journal of Mathematics, P.O. Box 969, Carmel Valley, CA 93924, U.S.A Old back numbers obtainable from Kraus Periodicals Co., Route 100, Millwood, NY 10546.

PUBLISHED BY PACIFIC JOURNAL OF MATHEMATICS, A NON-PROFIT CORPORATION

Printed at Kokusai Bunken Insatsusha (International Academic Printing Co., Ltd.). 8-8, 3-chome, Takadanobaba, Shinjuku-ku, Tokyo 160, Japan. 


\section{Pacific Journal of Mathematics}

\section{Vol. 91, No. 2 December, 1980}

Victor P. Camillo and Julius Martin Zelmanowitz, Dimension modules ... . . 249

Yonina S. Cooper, Stable sequences in pre-abelian categories ........... 263

Chandrakant Mahadeorao Deo and H. Ship-Fah Wong, On Berry-Esseen approximation and a functional LIL for a class of dependent random fields.........................................

H. P. Dikshit and S. N. Dubey, $|C, 1|$ summability of series associated with

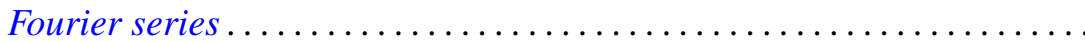

M. Edelstein, On the homomorphic and isomorphic embeddings of a semiflow into a radial flow.

Gilles Godefroy, Compacts de Rosenthal ..................... 293

James Guyker, Commuting hyponormal operators ................ 307

Thomas Eric Hall and Peter R. Jones, On the lattice of varieties of bands of

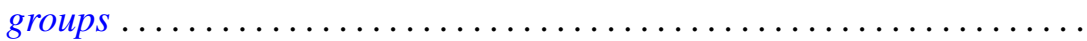

Taqdir Husain and Saleem H. Watson, Topological algebras with orthogonal Schauder bases ....................................

V. K. Jain, Some expansions involving basic hypergeometric functions of two variables. . .

Joe W. Jenkins, On group actions with nonzero fixed points ........... 363

Michael Ellsworth Mays, Groups of square-free order are scarce ........ 373

Michael John McAsey, Canonical models for invariant subspaces... 377

Peter A. McCoy, Singularities of solutions to linear second order elliptic partial differential equations with analytic coefficients by approximation methods...

Terrence Millar, Homogeneous models and decidability.

Stephen Carl Milne, A multiple series transformation of the very well poised

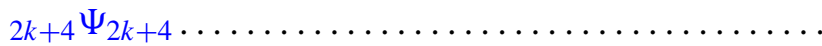

Robert Olin and James E. Thomson, Irreducible operators whose spectra are spectral sets...

Robert John Piacenza, Cohomology of diagrams and equivariant singular

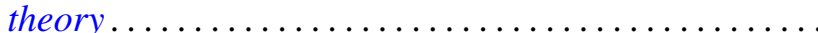

Louis Jackson Ratliff, Jr., Integrally closed ideals and asymptotic prime divisors

Robert Breckenridge Warfield, Jr., Cancellation of modules and groups and stable range of endomorphism rings.................

B. J. Day, Correction to: "Locale geometry" ...............

Stanley Stephen Page, Correction to: "Regular FPF rings" ... 